\title{
Trends and Determinants of Anemia among Under-Five Children in Ethiopia, Evidence from 2005 - 2016 Ethiopian Demographic and Health Survey; Mixed-Effect Binary Logistic Regression and Multivariate Decomposition Analysis
}

\author{
Abebew Aklog Asmare ${ }^{1 *} \quad$ Yitateku Adugna Agmas $^{2}$ \\ 1 Department Statistics, College of Natural and Computational Science, Mekdela Amba University, Tuluawlyia, \\ Ethiopia \\ 2 Department of Rural Development and Agricultural Extension, College Agriculture and Natural Resource, \\ Mekdela Amba University, Tuluawlyia, Ethiopia
}

\begin{abstract}
Background: Anemia is a major public health problem and it is defined as the condition of having a low number of red blood cells or a low amount of haemoglobin from the normal level. This situation touches most underfive-year old children. Objective: This study aimed to examine the trends of anemia prevalence among children aged between 6 to 59 months and its determinants in Ethiopia based on three consecutive Ethiopian Demographic and Health Surveys (EDHSs). Methods: A total of 3,716 in 2005, 8,365 in 2011 and 7,563 in 2016 children aged between 6 to 59 month were involved in this study. To identify significantly contributing factors for the increase in anemia prevalence in Ethiopia over the last 11 years' logit-based multivariate decomposition analysis was used and mixed-effect logistic regression model was fitted to identify determinant factors. Result: $47.13 \%$ of the change in anemia prevalence over time was attributable to difference in behavior. Being single $(\mathrm{AOR}=0.665 ; 95 \% \mathrm{CI}$ : $0.482-0.737)$, big stunted (AOR $=1.533 ; 95 \% \mathrm{CI}: 1.359-1.729)$, being underweight $(\mathrm{AOR}=1.200 ; 95 \% \mathrm{CI}: 1.040-1.384)$, being wasted $(\mathrm{AOR}=1.261 ; 95 \% \mathrm{CI}: 1.038-1.530)$, having more than three under-five children $(\mathrm{AOR}=1.463 ; 95 \% \mathrm{CI}: 1.041-2.058)$, source of drinking water was not improved $(\mathrm{AOR}=1.165 ; 95 \% \mathrm{CI}: 1.052-1.291)$ mother was anemic $(\mathrm{AOR}=1.664 ; 95 \% \mathrm{CI}: 1.487-1.862)$, fever history $(\mathrm{AOR}=1.427 ; 95 \% \mathrm{CI}$ : $1.211-1.683$ ) were more likely to experience the prevalence of anemia. Conclusion: This study found that the prevalence of anemia has increases over time in Ethiopia since 2005. Stunning, underweight, wasting, mothers age, source of drinking water, number of under-five children, wealth status, maternal anemia, birth order, being twin or single, age of children, birth size, fever and cough history were significant predictors of anemia prevalence. Designing interventions that address maternal anemia and strengthening the economic status of the family are recommended to reduce childhood anemia. Furthermore, it is better to strengthen the strategies of early detection and management of stunted, wasted, and underweight children to decrease childhood anemia.
\end{abstract}

Keywords: Childhood anemia, Mixed effect logistic regression, Multivariate decomposition analysis, Ethiopia DOI: $10.7176 / \mathrm{JCSD} / 67-03$

Publication date: January $31^{\text {st }} 2022$

\section{Background}

Anemia is a major public health problem [1]. It is defined as the condition of having a low number of red blood cells or a low amount of haemoglobin from the normal level [2-5]. This situation touches most under-five-year old children and pregnant women [6-8]. To address the full scope of causes of anemia the terms anemia, iron deficiency and iron-deficiency anemia (IDA are often used interchangeably [2]. According to World Health Organization [2] definition, anemia among under-five children as a $\mathrm{Hb}$ concentration below $110 \mathrm{~g} / \mathrm{L}$ were anemic [9]. For populations living at altitudes greater than $1000 \mathrm{~m}$ above sea level, as well as among smokers, it is recommended to make adjustments to these cut-off values [10].

Global burden of disease report in 2016 shows that, more than $27 \%$ of the world's population, nearly, 1.93 billion people were affected by anemia, developing and middle-income countries account for more than $89 \%$ of anemia cases, preschool children and reproductive-aged women are excessively affected by anemia [11]. Anemia among children is a public health problem globally [12]. In 2017, the World Health Organization [2] data source presented that the universal prevalence of anemia for under-five children was $41.7 \%$. Thus a problem is worse in Africa, $59.3 \%$ of children under five were anemic [4, 13]. Furthermore, the estimated prevalence of anemia in children aged 6-59 months is $62.3 \%$ in SSA approximately 84.5 million. This statistics put SSA as the region with the highest prevalence of anemia [14, 15]. Anemia has negative effects on physical development, attentiveness, memory, and academic performance of children $[16,17]$.

In Ethiopia, the prevalence of anemia in children less than 5 years is persistently higher than expected for the last two decades [18]. According to the respective Ethiopia Demographic and Health Survey reports, anemia prevalence among children 6 - 59 months of age increased from 44\% in 2011 to $57 \%$ in 2016 [19]. Even though 
the government of Ethiopia applied tremendous efforts, childhood anemia was a major public health problem according to WHO criteria. Many researches had been conducted to show its prevalence and associated factors [18].

Anemia level of mother $[4,12,18]$, number of under-five children [20-22], wealth status of the household $[4,14,23-25]$, mothers' age [4, 12, 18], source of drinking water [18, 26], gender of household head [27], birth size of child [25], being twin or single [4, 28], birth order of child [4], fever in the last two week [4, 12], cough in the last two week [4], age of children in month [14, 25-27, 29], stunting [4, 12, 21], underweight [4], wasting $[4,12]$ and region $[12,25]$.

However, some of the studies were derived from research conducted in specific localized groups which were not representative of the entire Ethiopia [5, 21, 28, 30,31], the rest were focused on the prevalence and associated factors of anemia prevalence only $[12,18,22,32]$ and failed to capture the trends and determinants of infant mortality in Ethiopia over time using a Logit based Multivariate Decomposition analysis for Non-linear Response Model. Therefore, this study aimed to investigate the trend and determinants of anemia prevalence among children aged between 6 and 59 months in Ethiopia over time. Understanding the trends and determinants of anemia prevalence could help public health planners, and partners to design evidence-based interventions to effectively reduce prevalence of anemia.

\section{Materials and Methods \\ Data Source and Population}

This study used 2005, 2011 and 2016 Ethiopian Demographic and Health surveys (EDHSs) data. These EDHSs are nationally representative cross-sectional surveys performed in 9 geographical regions and 2 city administrations for every five years. In each of EDHSs surveys, stratified two stage clusters sampling was accomplished. Stratification was realized by separating each region into urban and rural areas. Accordingly, a total of 21 sampling strata have been formed. In the first stage, a total of 540 Enumeration Areas (EAs) for EDHS 2005, and 645 EAs for EDHS 2016 were randomly selected proportional to the EA size. At the second, on average 27 to 32 households per EAs were selected [19, 33, 34].

\section{Ethical Consideration}

The data was accessed from the Measure DHS website after permission was approved through an online request by clarifying the objective of the study. The variables of the study were extracted from Kid Record (KR file) from each EDHSs data set. The complete procedure for sampling was designated in the detailed EDHS report $[19,33,34]$.

\section{Variables of the Study}

Variables reflected in this study were founded on literatures which have been conducted at the global level. The Possible determinant factors estimated to be associated with anemia status of under-five children were encompassed as variables of the study.

\section{Outcome Variable}

Hemoglobin is essential for transporting oxygen to tissues and organs in the body. Hemoglobin analysis was carried out onsite using a battery operated portable HemoCue analyzer. Parents of children with a hemoglobin level under $11 \mathrm{~g} / \mathrm{dl}$ were instructed to take the child to a health facility for follow-up care. Unadjusted hemoglobin values are gotten using the HemoCue tool. Given that hemoglobin requirements differ substantially depending on altitude, a modification to sea-level equivalents has been made before classifying children by level of anemia. Occurrence of anemia, based on hemoglobin levels is adjusted for altitude by hemoglobin in grams per decilitre (g/dl) [35]. The outcome variable of this study was anemia status of children aged 6-59 months. For the current analysis, response variable (anemia status) was dichotomized indicating not anemic coded by zero or anemic coded by 1 .

$$
Y_{i j}=\left\{\begin{array}{c}
\text { Not anemic }=0 \\
\text { Anemic }=1
\end{array}\right.
$$

\section{Explanatory variables}

Region of the study participants, mothers 'age, number of under-five children in the household, source of drinking water, sex of household head, wealth status of the household, anemia level of mother, birth order of children, being single or twin, age of children, birth size of children, fever in the last two weak preceding the survey, cough in the last two week preceding the survey, stunting, underweight and wasting status of children were considered as independent variables for this study. 


\section{Handling Mechanisms of Missing Data}

Missing data is a common problem of most survey data. Missing data presents a difficult in statistical data analyses. The first issue in dealing with the problem of missing data by determining the missing data mechanism. Three types of missing data mechanism were distinguished by [36]; among them we apply missing completely at random (MCAR) that is missingness is not linked to the variables under study. To handle missing value, we used list wise deletion method which is a common approach and easy to perform by deleting all incomplete observations from the analysis.

\section{Statistical Analysis}

The data were taken from the KID Record (KR file) of each EDHSs data sets. Before starting any statistical analysis, the data were weighted using sampling weight for probability sampling and non-response to restore the representativeness of the survey and get reliable statistical estimates, the data analysis was executed using STATA 16. The data analysis includes three important parts. First, descriptive statistics and trends were analyzed by each characteristics of variables among all children aged $6-59$ months. Second, to understand the extent to which each selected covariate contributed to the observed trend in anemia prevalence, multivariate decomposition procedures were implemented finally, multilevel binary logistic regression analysis was carried out to identify factors associated with prevalence of anemia among children aged $6-59$ months in Ethiopia.

\section{Trend and decomposition analysis}

The trend phase was divided into three periods; first period (2011 - 2005), second period (2016 - 2011) and third phase or over all phase (2016 - 2005) to see the differences in magnitude of anemia over time based on different characteristics. The trend was measured using descriptive analyses stratified by different characteristics and was measured separately for each phases 2011 - 2005, 2016-2011 and 2016-2005. Multivariate decomposition provides a way to analyze differences in the outcome between two surveys or between two points of time (comparable 2005 and 2016 survey years). The decomposition procedure divides the total increment in prevalence of anemia into two portions; the portion that can be attributed to the change in composition or the prevalence of a set of indicators (referred to as the endowments portion) and the portion that can be attributed to the change in the effect of these indicators (referred to as the coefficient portion). Therefore, the observed change in load of anemia between two points of time was additively decomposed in to endowment (characteristics) component and coefficient (effect of characteristics) component. The analysis was implemented using recently developed mvdcmp Stata package [37]. The equation is given by

$\Delta Y^{i-j}=\left(X^{i}-X^{j}\right) \beta^{i}+X^{j}\left(\beta^{i}-\beta^{j}\right), i \neq j$

Where $i, j=2005,2011$ and $2016 \Delta Y$ is the difference in mean prediction of anemia prevalence between year $i$ and year $j$, given that of different characteristics of X. $\beta$ is the estimated regression coefficients. $\left(X^{i}-X^{j}\right) \beta^{i}$ represents the difference due to endowment between the $i^{\text {th }}$ and $j^{\text {th }}$ years. $X^{j}\left(\beta^{i}-\beta^{j}\right)$ represents the difference due to coefficients between the $i^{\text {th }}$ and $j^{\text {th }}$ years.

\section{Determinants of anemia prevalence among children aged 6 -59 months}

As the data used for this study had nested structure, children aged $6-59$ months within the same cluster share similar characteristics than children aged $6-59$ months out of the given cluster. In hierarchical data, most popular statistical methods such as mixed-effect regression analysis is used to get reliable estimate. Thus, a twolevel mixed-effect logistic regression model (both fixed and random effect) was fitted using enumeration areas as a random variable to draw a valid conclusion. The assumptions of the mixed-effect binary logistic regression model were tested using the Intra-class Correlation Coefficient (ICC) and Likelihood Ratio (LR) test, whereas Median Odds Ratio [27] and Proportional Change in Variance (PCV) were calculated to quantity the deviation across clusters. ICC measures the degree of heterogeneity of anemia prevalence between clusters.

$$
I C C=\frac{\sigma^{2}}{\left(\sigma^{2}+\frac{\pi}{3}\right)}
$$

The MOR measures the between cluster variation in terms of odds ratio. The median value of the odds ratio between the cluster at high risk of anemia prevalence and at lower risk of anemia prevalence when randomly picking out two clusters (EAs).

$M O R=(0.95 * \sigma)$

[4]

$\sigma$ indicates that cluster standard deviation

PCV measures the total variation in anemia prevalence explained by the final model compared to the null model. $P C V=\frac{\text { var }(\text { null model })-\operatorname{var}(\text { full model })}{\operatorname{var}(\text { null model })}$

Bayesian Information Criteria (BIC), Akaike Information Criteria (AIC), and deviance were used for model evaluation. Models having lower deviance was chosen as a nested model. We identified the independent 
variables based on previous literature conducted on determinants of anemia prevalence among children aged $6-$ 59 months. The Adjusted Odds Ratio [27] with a 95\% Confidence Interval (CI) and p-value $<0.05$ in the multivariable model were used to declare significant determinant factors of anemia prevalence among children aged 6 -59 months.

\section{Results}

\section{Characteristics of the study population}

Table 1 presents the percentage distribution of anemia in children aged $6-59$ months based on children's maternal and household characteristics reports from 2005 - 2016 EDHS. Thus, the analysis encompassed weighted data from 3,716 in the 2005, 8,365 in the 2011, and 7,613 children in the 2016 EDHS.

Established on demographic characteristics of EDHSs data, more than $6 \%$ of the households were urban settlers; $6.2 \%$ in 2005, 11.8\% in 2011 and 9.9 in 2016. More than 86\%; 88.1 in 2005, 86.6\% in 2011 and $90.2 \%$ in 2016 of the total households in three EDHSs survey were leaded by males. Regarding to birth characteristics of EDHSs data, the highest percentage (2.4\%) of twin birth was in 2016. Highest and lowest percentage of small birth size (29\%) and (24.8\%) was reported in 2011 and 2016 respectively. Regrading to birth order of children, highest proportion of children have birth order of 5 and more in all three EDHSs survey; $42.7 \%$ in $2005,38.1 \%$ in 2011 and $27.6 \%$ in 2016. Amongst the surveyed households, $65.3 \%, 80.0 \%$ and 52.3 of households were drink not improved water in 2005, 2011 and 2016 EDHSs survey period respectively. Regarding to households' wealth status $13.6 \%$ of the households in $2005,14.1 \%$ in 2011 and $13.4 \%$ in 2016 were richest. From the total surveyed population $98.4 \%$ households in $2005,98.3$ in 2011 and $97.5 \%$ in 2016 have one to three children. $54.5 \%$ of household in $2005,52.9 \%$ in 2011 and $50.5 \%$ of household in 2016 have 5 to 7 size per household.

Table 1 Frequency and Percentage distribution of characteristics of respondents and their children in Ethiopia

\begin{tabular}{|c|c|c|c|c|}
\hline \multirow[t]{2}{*}{ Variable } & \multirow[t]{2}{*}{ Characteristics } & \multicolumn{3}{|c|}{ Frequency and percentage in EDHS Periods } \\
\hline & & $\begin{array}{l}\text { EDHS } 2005 \\
\text { Weighted } \\
\text { frequency }(\%)\end{array}$ & $\begin{array}{l}\text { EDHS } 2011 \\
\text { Weighted } \\
\text { frequency }(\%)\end{array}$ & $\begin{array}{l}\text { EDHS } 2016 \\
\text { Weighted } \\
\text { frequency }(\%)\end{array}$ \\
\hline $\begin{array}{c}\text { Prevalence of } \\
\text { children anemia }\end{array}$ & $\begin{array}{l}\text { Not Anemic } \\
\text { Anemic }\end{array}$ & $\begin{array}{l}1710(46.0) \\
2006(54.0)\end{array}$ & $\begin{array}{l}4644(55.5) \\
3721(44.5)\end{array}$ & $\begin{array}{l}3288(42.8) \\
4325(57.2)\end{array}$ \\
\hline $\begin{array}{c}\text { Age of mother in } \\
\text { years }\end{array}$ & $\begin{array}{l}15-24 \\
25-34 \\
35-49\end{array}$ & $\begin{array}{l}816(22.0) \\
1850(49.8) \\
1050(28.3)\end{array}$ & $\begin{array}{l}1863(22.3) \\
4443(53.1) \\
2059(24.6)\end{array}$ & $\begin{array}{l}1535(20.3) \\
4121(54.5) \\
1906(25.2)\end{array}$ \\
\hline $\begin{array}{l}\text { Source of drinking } \\
\text { water }\end{array}$ & $\begin{array}{l}\text { Not Improved } \\
\text { Improved }\end{array}$ & $\begin{array}{l}2426(65.3) \\
1291(34.7)\end{array}$ & $\begin{array}{l}6688(80.0) \\
1677(20.0)\end{array}$ & $\begin{array}{l}3952(52.3) \\
3611(47.7) \\
\end{array}$ \\
\hline $\begin{array}{l}\text { Number of under } \\
\text { five children }\end{array}$ & $\begin{array}{l}1-3 \\
4 \text { and above }\end{array}$ & $\begin{array}{l}3659(98.4) \\
58(1.6)\end{array}$ & $\begin{array}{l}8223(98.3) \\
142(1.7)\end{array}$ & $\begin{array}{l}7373(97.5) \\
190(2.5)\end{array}$ \\
\hline $\begin{array}{c}\text { Sex of household } \\
\text { head }\end{array}$ & $\begin{array}{l}\text { Male } \\
\text { Female }\end{array}$ & $\begin{array}{l}3276(88.1) \\
441(11.9)\end{array}$ & $\begin{array}{l}7245(86.6) \\
1120(13.4) \\
\end{array}$ & $\begin{array}{l}6871(90.2) \\
742(9.8)\end{array}$ \\
\hline Wealth Status & $\begin{array}{l}\text { Lowest } \\
\text { Lower } \\
\text { Middle } \\
\text { Higher } \\
\text { Highest }\end{array}$ & $\begin{array}{l}829(22.3) \\
793(21.3) \\
832(22.4) \\
755(20.3) \\
506(13.6)\end{array}$ & $\begin{array}{l}1907(22.8) \\
1877(22.4) \\
1721(20.6) \\
1683(20.1) \\
1177(14.1) \\
\end{array}$ & $\begin{array}{l}1723(22.8) \\
1778(23.5) \\
1660(21.9) \\
1388(18.4) \\
1013(13.4) \\
\end{array}$ \\
\hline $\begin{array}{l}\text { Anemia level of } \\
\text { mother }\end{array}$ & $\begin{array}{l}\text { Not Anemic } \\
\text { Anemic }\end{array}$ & $\begin{array}{l}2655(71.4) \\
1062(28.6) \\
\end{array}$ & $\begin{array}{l}6866(82.4) \\
1498(17.9) \\
\end{array}$ & $\begin{array}{l}5281(69.8) \\
2282(30.2) \\
\end{array}$ \\
\hline Birth order & $\begin{array}{l}\text { First } \\
\text { Second } \\
3-4 \\
5 \text { and above }\end{array}$ & $\begin{array}{l}535(14.4) \\
588(15.8) \\
1006(27.1) \\
1587(42.7) \\
\end{array}$ & $\begin{array}{l}1463(17.5) \\
1428(17.1) \\
2291(27.4) \\
3183(38.1) \\
\end{array}$ & $\begin{array}{l}1241(16.4) \\
1222(16.2) \\
2084(27.6) \\
3015(39.9) \\
\end{array}$ \\
\hline Birth type & $\begin{array}{l}\text { Single } \\
\text { Multiple }\end{array}$ & $\begin{array}{l}3673(98.8) \\
43(1.2) \\
\end{array}$ & $\begin{array}{l}8199(98.0) \\
166(2.0) \\
\end{array}$ & $\begin{array}{l}7380(97.6) \\
182(2.4) \\
\end{array}$ \\
\hline $\begin{array}{l}\text { Age of child in } \\
\text { months }\end{array}$ & $\begin{array}{l}6-11 \\
12-23 \\
24-59\end{array}$ & $\begin{array}{l}397(10.7) \\
812(21.9) \\
2507(67.5) \\
\end{array}$ & $\begin{array}{l}936(11.2) \\
1691(20.2) \\
5738(68.6) \\
\end{array}$ & $\begin{array}{l}881(11.7) \\
1691(22.4) \\
4991(66.0) \\
\end{array}$ \\
\hline $\begin{array}{l}\text { Size of child at } \\
\text { birth }\end{array}$ & $\begin{array}{l}\text { Small } \\
\text { Average } \\
\text { Large }\end{array}$ & $\begin{array}{l}989(26.6) \\
1483(39.9) \\
1244(33.5)\end{array}$ & $\begin{array}{l}2426(29.0) \\
3270(39.1) \\
2669(31.9)\end{array}$ & $\begin{array}{l}1876(24.8) \\
3227(42.7) \\
2459(32.5)\end{array}$ \\
\hline
\end{tabular}




\begin{tabular}{|l|l|l|l|l|}
\hline Fever & No & $2999(80.7)$ & $6929(82.8)$ & $6615(87.5)$ \\
& Yes & $717(19.3)$ & $1436(17.2)$ & $1117(14.8)$ \\
\hline Cough & No & $3031(81.6)$ & $6688(80.0)$ & $6038(79.8)$ \\
& Yes & $685(18.4)$ & $1676(20)$ & $1525(20.2)$ \\
\hline Stunting & No & - & $4311(51.5)$ & $4406(58.3)$ \\
& Yes & - & $4053(48.5)$ & $3156(41.7)$ \\
\hline Underweight & No & & $5758(68.8)$ & $5650(74.7)$ \\
& Yes & - & $2607(31.3)$ & $1913(25.3)$ \\
\hline Wasting & No & $7580(90.6)$ & $6844(90.5)$ \\
& Yes & $269(7.2)$ & $785(9.4)$ & $718(9.5)$ \\
\hline & Tigray & $31(0.8)$ & $570(6.8)$ & $488(6.4)$ \\
& Afar & $794(21.4)$ & $81(1.0)$ & $75(1.0)$ \\
& Amhara & $1525(41.0)$ & $3600(43.0)$ & $1466(19.4)$ \\
& Oromia & $115(3.1)$ & $207(2.5)$ & $3301(43.6)$ \\
& Somali & $36(1.0)$ & $95(1.1)$ & $81(1.1)$ \\
& Benishangul & $884(23.8)$ & $1817(21.7)$ & $1647(21.8)$ \\
& SNNP & $9(0.2)$ & $25(0.3)$ & $17(0.2)$ \\
& Gambela & \multicolumn{3}{|l|}{} \\
\hline & Harari & $6(0.2)$ & $17(0.2)$ & $14(0.2)$ \\
& Addis Ababa & $36(1.0)$ & $132(1.6)$ & $139(1.8)$ \\
& Dire Dewa & $12(0.3)$ & $25(0.3)$ & $27(0.4)$ \\
\hline
\end{tabular}

Overall trends of anemia prevalence among children aged 6 - 59 in Ethiopia

By looking the trend, Ethiopia has been shown a decrement in anemia prevalence among under-five children from 2005 - 2011 and an increment from 2005 - 2016 and 2011 - 2016 survey period. Accordingly, it was 54\% in 2005 decreases by $9.5 \%$ in 2011 and increases by $3.2 \%$ in 2016 . The highest decrement was noticed in the first phase $(2011$ - 2005) with a 9.5\%-point change and the highest increment was in the second phase (2016 2011 ) with $12.7 \%$ point change. The overall change (2016-2005) in anemia prevalence was $3.2 \%$ point change increment (Fig. 1)

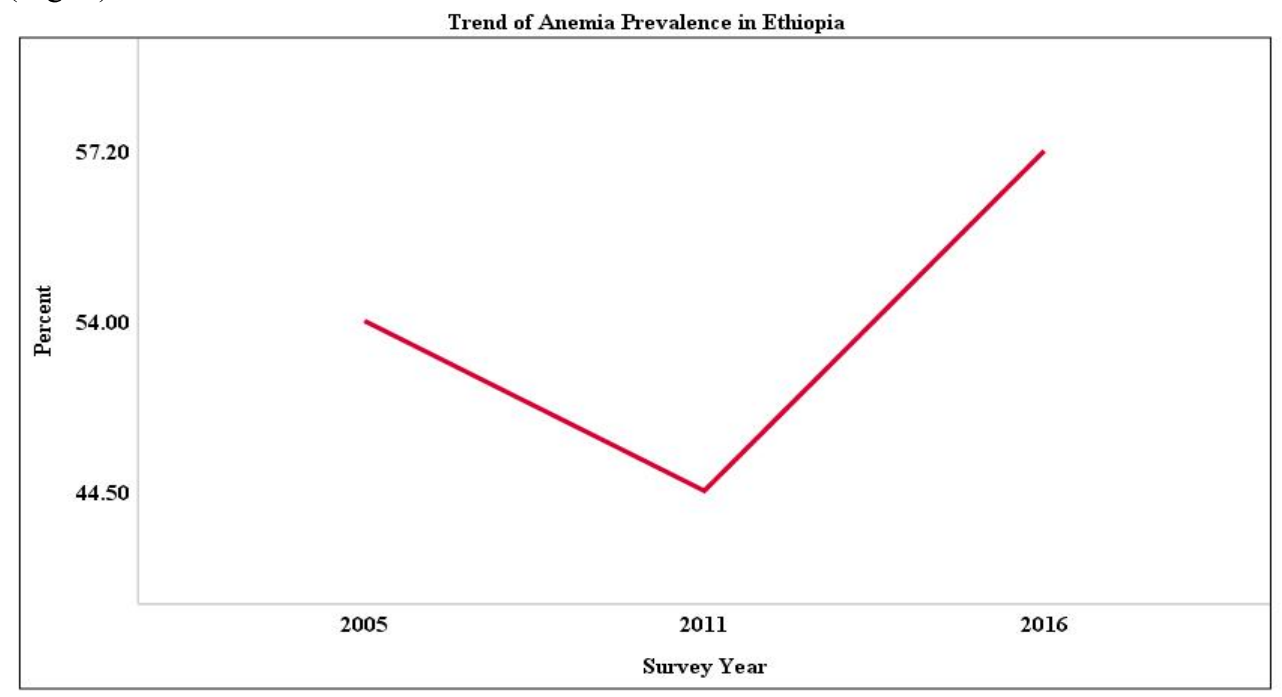

Fig. 1 Trend of Anemia Prevalence in Ethiopia from 2005 to 2016

Trends of anemia prevalence in Ethiopia by region, 2005 - 2016

Table 2 presents the Trends of anemia prevalence of children aged $6-59$ months in Ethiopia based on maternal and household characteristics from 2005 - 2016 EDHS.

The trends of anemia prevalence among children $6-59$ months aged revealed that variation based on different characteristics. anemia prevalence decrement was observed in most of the characteristics in the first phase $(2011$ - 2005) and increment in some of the characteristics, but anemia prevalence increment was observed in most characteristics at second phase and overall phase; some characteristics show a decrement. Among mothers aged $35-49$, the largest decrement was observed during the first phase of the study period $(2011-2005)$ with $11.8 \%$ point change followed by the overall phase $(2016-2005) 2.2 \%$ point change, but an 
increment in the second phase $(2016-2011)$ which is $9.6 \%$ point change. Based on region, the largest decrement in first phase was observed in Tigray regional state with $18.7 \%$ point change followed by Amhara with $16.2 \%$ point change and the largest increment was observed in Afar with 16.0\% point change followed by Dire Dewa with $0.4 \%$ point change. But in the second phase, anemia prevalence was increased all regions except Benishangul Gumz (Fig. 2). Similarly, in third phase anemia prevalence show decrement in Tigray, Amhara, Somali and Gambela whereas it shows an increment from the rest region. Households that have 4 and more than 4 under-five age children showed an increment in anemia prevalence in all phases; $16 \%, 23.1 \%$ and $39.1 \%$ point change respectively. Respondents who had have improved drinking water source showed decrement of anemia prevalence among under-five children in first phase.

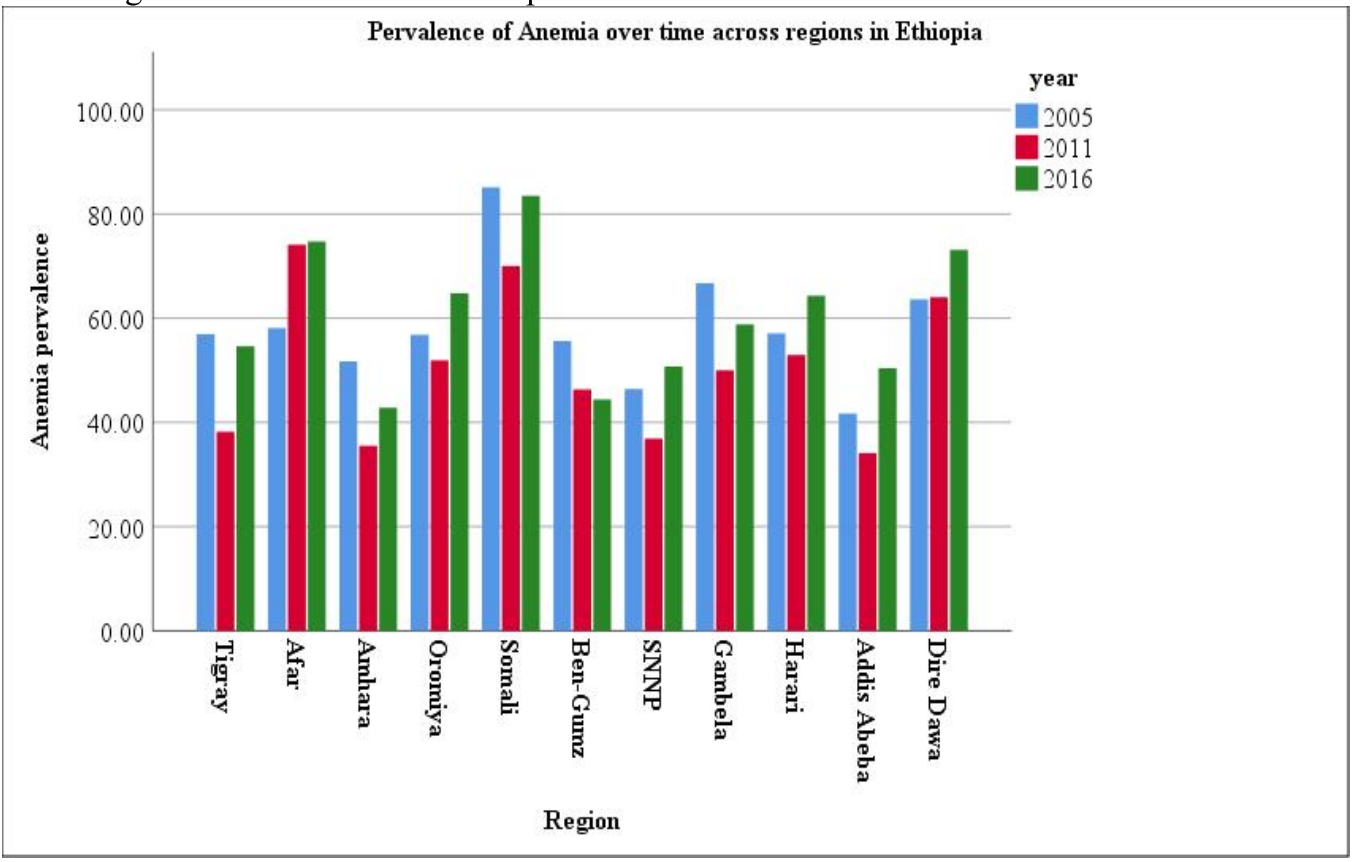

Fig. 2 Trend of prevalence of anemia over time across regions in Ethiopia 2005, 2011, 2016

Table 2 Trends of Anemia prevalence among under-five children by selected characteristics in Ethiopia.

\begin{tabular}{|c|c|c|c|c|c|c|c|}
\hline \multirow[t]{2}{*}{ Variable } & \multirow[t]{2}{*}{ Characteristics } & \multicolumn{3}{|c|}{ EDHSs periods } & \multicolumn{3}{|c|}{$\begin{array}{l}\text { Point difference in anemia } \\
\text { prevalence }\end{array}$} \\
\hline & & $\begin{array}{l}2005 \\
(2138)\end{array}$ & $\begin{array}{c}2011 \\
(5140)\end{array}$ & $\begin{array}{c}2016 \\
(8504)\end{array}$ & $\begin{array}{c}\text { Phase I } \\
(2011-2005)\end{array}$ & $\begin{array}{c}\text { Phase II } \\
(2016- \\
2011)\end{array}$ & $\begin{array}{c}\text { Phase III } \\
(2016- \\
2005) \\
\end{array}$ \\
\hline Age of mother & $\begin{array}{l}15-24 \\
25-34 \\
35-49\end{array}$ & $\begin{array}{l}57.5 \\
53.3 \\
54.3 \\
\end{array}$ & $\begin{array}{l}46.9 \\
44.4 \\
42.5\end{array}$ & $\begin{array}{l}63.3 \\
57.2 \\
52.1\end{array}$ & $\begin{array}{l}-10.6 \\
-8.9 \\
-11.8\end{array}$ & $\begin{array}{l}16.4 \\
12.8 \\
9.6\end{array}$ & $\begin{array}{r}5.8 \\
3.9 \\
-2.2 \\
\end{array}$ \\
\hline $\begin{array}{l}\text { No. of under- } \\
\text { five child }\end{array}$ & $\begin{array}{c}0-3 \\
4 \text { and above }\end{array}$ & $\begin{array}{l}54.4 \\
29.8\end{array}$ & $\begin{array}{l}44.5 \\
45.8\end{array}$ & $\begin{array}{l}56.9 \\
68.9\end{array}$ & $\begin{array}{l}-9.9 \\
16.0\end{array}$ & $\begin{array}{l}12.4 \\
23.1\end{array}$ & $\begin{array}{c}2.5 \\
39.1\end{array}$ \\
\hline Sex of $\mathrm{HHH}$ & $\begin{array}{c}\text { Male } \\
\text { Female }\end{array}$ & $\begin{array}{l}54.0 \\
54.1\end{array}$ & $\begin{array}{l}44.5 \\
44.2\end{array}$ & $\begin{array}{l}56.4 \\
64.2 \\
\end{array}$ & $\begin{array}{l}-9.5 \\
-9.9 \\
\end{array}$ & $\begin{array}{l}11.9 \\
13.0\end{array}$ & $\begin{array}{l}2.4 \\
3.1 \\
\end{array}$ \\
\hline Wealth Status & $\begin{array}{l}\text { Lowest } \\
\text { Lower } \\
\text { Middle } \\
\text { Higher } \\
\text { Highest }\end{array}$ & $\begin{array}{l}60.1 \\
55.9 \\
52.8 \\
49.7 \\
49.3 \\
\end{array}$ & $\begin{array}{l}48.3 \\
47.1 \\
43.8 \\
43.4 \\
36.7 \\
\end{array}$ & $\begin{array}{l}68.5 \\
57.1 \\
53.9 \\
54.3 \\
47.5 \\
\end{array}$ & $\begin{array}{l}-11.8 \\
-8.8 \\
-9.0 \\
-6.3 \\
-12.6 \\
\end{array}$ & $\begin{array}{c}20.2 \\
1.2 \\
10.1 \\
10.9 \\
10.8 \\
\end{array}$ & $\begin{array}{c}8.4 \\
-7.6 \\
1.1 \\
4.6 \\
-1.8 \\
\end{array}$ \\
\hline $\begin{array}{l}\text { Mother anemia } \\
\text { level }\end{array}$ & $\begin{array}{l}\text { Not anemic } \\
\text { Anemic }\end{array}$ & $\begin{array}{l}50.7 \\
62.1\end{array}$ & $\begin{array}{l}42.2 \\
54.7\end{array}$ & $\begin{array}{l}52.3 \\
68.6\end{array}$ & $\begin{array}{l}-8.5 \\
-7.4 \\
\end{array}$ & $\begin{array}{l}11.1 \\
13.9\end{array}$ & $\begin{array}{l}2.6 \\
6.5\end{array}$ \\
\hline $\begin{array}{l}\text { Birth order of } \\
\text { child }\end{array}$ & $\begin{array}{l}\text { First } \\
\text { Second } \\
\text { Three to four } \\
5 \text { and above }\end{array}$ & $\begin{array}{l}53.4 \\
50.4 \\
56.9 \\
53.7\end{array}$ & $\begin{array}{l}41.5 \\
43.4 \\
44.4 \\
46.4\end{array}$ & $\begin{array}{l}56.0 \\
55.1 \\
56.4 \\
59.0\end{array}$ & $\begin{array}{l}-11.9 \\
-7.0 \\
-12.5 \\
-7.3\end{array}$ & $\begin{array}{l}14.5 \\
11.7 \\
12.0 \\
12.6\end{array}$ & $\begin{array}{l}2.6 \\
4.7 \\
-0.5 \\
5.3\end{array}$ \\
\hline Birth type & $\begin{array}{l}\text { Single } \\
\text { Multiple }\end{array}$ & $\begin{array}{l}54.0 \\
48.8\end{array}$ & $\begin{array}{l}44.4 \\
48.5\end{array}$ & $\begin{array}{l}57.3 \\
51.9\end{array}$ & $\begin{array}{l}-9.6 \\
-0.3\end{array}$ & $\begin{array}{l}12.9 \\
3.4\end{array}$ & $\begin{array}{l}3.3 \\
3.1\end{array}$ \\
\hline
\end{tabular}




\begin{tabular}{|c|c|c|c|c|c|c|c|}
\hline Age of child & $\begin{array}{l}6-11 \\
12-23 \\
24-59\end{array}$ & $\begin{array}{l}76.9 \\
69.3 \\
45.4\end{array}$ & $\begin{array}{l}66.5 \\
58.2 \\
36.9 \\
\end{array}$ & $\begin{array}{l}76.3 \\
69.4 \\
49.6\end{array}$ & $\begin{array}{l}-10.4 \\
-11.1 \\
-8.5\end{array}$ & $\begin{array}{l}9.8 \\
11.2 \\
12.7\end{array}$ & $\begin{array}{l}-0.6 \\
0.1 \\
4.2\end{array}$ \\
\hline $\begin{array}{l}\text { Size of child at } \\
\text { birth }\end{array}$ & $\begin{array}{l}\text { Small } \\
\text { Average } \\
\text { Large }\end{array}$ & $\begin{array}{l}55.9 \\
52.4 \\
54.3\end{array}$ & $\begin{array}{l}45.7 \\
42.3 \\
46.1\end{array}$ & $\begin{array}{l}61.9 \\
54.5 \\
57.1\end{array}$ & $\begin{array}{l}-10.2 \\
-10.1 \\
-8.2\end{array}$ & $\begin{array}{l}16.2 \\
12.2 \\
11.0\end{array}$ & $\begin{array}{l}6.0 \\
2.1 \\
2.8\end{array}$ \\
\hline Fever & $\begin{array}{l}\text { No } \\
\text { Yes }\end{array}$ & $\begin{array}{l}54.0 \\
54.1\end{array}$ & $\begin{array}{l}43.4 \\
49.9\end{array}$ & $\begin{array}{l}55.8 \\
64.9\end{array}$ & $\begin{array}{l}-10.6 \\
-4.2\end{array}$ & $\begin{array}{l}12.4 \\
15.0\end{array}$ & $\begin{array}{l}1.4 \\
10.8\end{array}$ \\
\hline Cough & $\begin{array}{l}\text { No } \\
\text { Yes }\end{array}$ & $\begin{array}{l}54.8 \\
50.4\end{array}$ & $\begin{array}{l}44.0 \\
46.4\end{array}$ & $\begin{array}{l}56.5 \\
59.9\end{array}$ & $\begin{array}{l}-10.8 \\
-4.0\end{array}$ & $\begin{array}{l}12.5 \\
13.5\end{array}$ & $\begin{array}{l}1.7 \\
9.5\end{array}$ \\
\hline Stunting & $\begin{array}{l}\text { No } \\
\text { Yes }\end{array}$ & - & $\begin{array}{l}41.9 \\
47.2 \\
\end{array}$ & $\begin{array}{l}54.3 \\
61.2 \\
\end{array}$ & - & $\begin{array}{l}12.4 \\
14.0\end{array}$ & - \\
\hline Wasting & $\begin{array}{l}\text { No } \\
\text { Yes }\end{array}$ & - & $\begin{array}{l}43.1 \\
57.6\end{array}$ & $\begin{array}{l}56.0 \\
68.1\end{array}$ & - & $\begin{array}{l}12.9 \\
10.5\end{array}$ & - \\
\hline Underweight & $\begin{array}{l}\text { No } \\
\text { Yes } \\
\end{array}$ & - & $\begin{array}{l}41.3 \\
51.6 \\
\end{array}$ & $\begin{array}{l}54.9 \\
64.0 \\
\end{array}$ & - & $\begin{array}{l}13.6 \\
12.4 \\
\end{array}$ & - \\
\hline Region & $\begin{array}{l}\text { Tigray } \\
\text { Afar } \\
\text { Amhara } \\
\text { Oromo } \\
\text { Somali } \\
\text { B/Gumz } \\
\text { SNNP } \\
\text { Gambela } \\
\text { Harari } \\
\text { Addis Ababa } \\
\text { Dire Dewa }\end{array}$ & $\begin{array}{l}56.9 \\
58.1 \\
51.7 \\
56.8 \\
85.1 \\
55.6 \\
46.4 \\
66.7 \\
57.1 \\
41.7 \\
63.6\end{array}$ & $\begin{array}{l}38.2 \\
74.1 \\
35.5 \\
51.9 \\
70.0 \\
46.3 \\
36.9 \\
50.0 \\
52.9 \\
34.1 \\
64.0\end{array}$ & $\begin{array}{l}54.6 \\
74.7 \\
42.8 \\
64.8 \\
83.5 \\
44.4 \\
50.7 \\
58.8 \\
64.3 \\
50.4 \\
73.1\end{array}$ & $\begin{array}{l}-18.7 \\
16.0 \\
-16.2 \\
-4.9 \\
-15.1 \\
-9.3 \\
-9.5 \\
-16.7 \\
-4.2 \\
-7.6 \\
0.4\end{array}$ & $\begin{array}{l}16.4 \\
0.6 \\
7.3 \\
12.9 \\
13.5 \\
-1.9 \\
13.8 \\
8.8 \\
11.4 \\
16.3 \\
13.1\end{array}$ & $\begin{array}{l}-2.3 \\
16.6 \\
-8.9 \\
8.0 \\
-1.6 \\
-12.1 \\
4.3 \\
-7.9 \\
7.2 \\
8.7 \\
13.5\end{array}$ \\
\hline
\end{tabular}

Decomposition analysis

Table 3 Overall multivariate decomposition analysis of anemia prevalence in Ethiopia, 2005-2016

\begin{tabular}{|l|l|l|l|}
\hline Prevalence of anemia & Cofe. & $\mathbf{9 5} \%$ CI. & Pct. \\
\hline E & 0.0248 & $(0.0231-0.0282)$ & 47.13 \\
\hline C & 0.0278 & $(0.0074-0.0482)$ & 52.87 \\
\hline R & 0.0525 & $(0.0326-0.0724)$ & \\
\hline
\end{tabular}

Table 3 presents the overall decomposition result of anemia prevalence of children aged $6-59$ months in Ethiopia based on maternal and household characteristics from 2005 - 2016 EDHS.

Overall from 2005 to 2016, there has been a significant increment in prevalence of anemia in Ethiopia. The overall decomposition result showed that $47.13 \%$ of increment in prevalence of anemia over time was due to behavioral changes between the surveys. About $52.87 \%$ of increment was due to difference in characteristics (population proportion).

Factors birth order of child and fever showed a significant effect for the decline of anemia prevalence, whereas factors including number of under-five children in a family, wealth status of a family and mother's anemia status showed a significant effect for the increment of anemia prevalence. Keeping compositional changes constant, change in behavior of birth order of children who are got first birth contributed $3.43 \%$ for the decline of anemia prevalence for the last eleven years as compared to children who had got $5^{\text {th }}$ and more birth. Compared with children who had got fifth and more birth, behavioral change of children who had got second birth were contributes $0.78 \%$ for decrement anemia prevalence over time. Behavioral change of households who had lowest wealth status were contributed $1.68 \%$ for the increment of anemia prevalence for the last eleven years as compared to households who had highest wealth status. Similarly, the behavioral households having four and more than four under-five children contributing $2.0 \%$ for the increment of anemia prevalence for the last eleven years as compared to households having less than four under-five children (Table 4). 
Table 4 The detailed multivariate decomposition analysis of anemia prevalence in Ethiopia, 2005-2016

\begin{tabular}{|l|l|l|l|l|}
\hline \multirow{2}{*}{$\begin{array}{l}\text { Anemia } \\
\text { prevalence }\end{array}$} & Difference due to characteristics (E) & \multicolumn{2}{l|}{ Difference due to coefficients (C) } \\
\cline { 2 - 5 } & Cofe. With 95\% CI and P-value & Pct. & \multicolumn{1}{l|}{ Cofe. With 95\% CI and P-value } & Pct. \\
\hline Age of mother & Ref. & & Ref. & \\
\hline $15-23$ & $-0.0015(-0.0025,-0.0005), 0.004^{*}$ & -2.77 & $-0.0122(-0.0415,0.017), 0.411$ & -23.32 \\
\hline $24-34$ & $0.0035(0.0022,0.0047),<0.001^{*}$ & 6.60 & $-0.033(-0.0539,-0.1207), 0.002^{*}$ & -62.81 \\
\hline $35-49$ & \multicolumn{5}{|l}{} \\
\hline Number of under-five Children & & Ref. & 7.02 \\
\hline $1-3$ & Ref. & 3.17 & $0.0037(0.0015,0.0059), 0.001^{*}$ & \\
\hline 4 and above & $0.0017(0.0007,0.0026), 0.001^{*}$ &
\end{tabular}

\begin{tabular}{|c|c|c|c|c|}
\hline \multirow{2}{*}{$\begin{array}{l}\text { Anemia } \\
\text { prevalence }\end{array}$} & \multicolumn{2}{|l|}{ Difference due to characteristics (E) } & \multicolumn{2}{|l|}{ Difference due to coefficients $(\mathrm{C})$} \\
\hline & Cofe. With $95 \%$ CI and P-value & Pet. & Cofe. With $95 \%$ CI and P-value & Pct. \\
\hline \multicolumn{5}{|c|}{ Gender of household head } \\
\hline Male & Ref. & & Ref. & \\
\hline Female & $0.0011(0.0003,0.0018), 0.005$ & 3.17 & $0.0083(0.0006,0.0159), 0.033^{*}$ & 15.75 \\
\hline \multicolumn{5}{|c|}{ Wealth status of the household } \\
\hline Poorest & Ref. & & Ref. & \\
\hline Poorer & $0.0009(0.0006,0.0012),<0.001^{*}$ & 1.68 & $-0.0094(-0.0202,0.0013), 0.084$ & -17.99 \\
\hline Middle & $0.0067(0.0052,0.0083),<0.001^{*}$ & 12.81 & $-0.0134(-0.2453,-0.0023), 0.018^{*}$ & -25.53 \\
\hline Higher & $0.0070(0.0053,0.0087),<0.001^{*}$ & 13.28 & $-0.1374(-0.0242,-0.0033), 0.010^{*}$ & -26.17 \\
\hline Highest & $-0.0012(-0.0015,-0.0009)<0.001^{*}$ & -2.24 & $-0.0036(-0.1362,0.0064), 0.477$ & -6.91 \\
\hline \multicolumn{5}{|c|}{ Anemia status of mother } \\
\hline Not anemic & Ref. & & Ref. & \\
\hline Anemic & $0.0063(0.0052,0.0073),<0.001$ & 11.91 & $0.0106(-0.0017,0.0229), 0.092$ & 20.15 \\
\hline \multicolumn{5}{|c|}{ Birth order of children } \\
\hline First & Ref. & & Ref. & \\
\hline Second & $-0.0018(-0.0026,-0.001),<0.001^{*}$ & -3.43 & $-0.0135(-0.0254,-0.0016), 0.026^{*}$ & -25.75 \\
\hline 3 to 4 & $-0.0004(-0.0006,-0.0002),<0.001^{*}$ & -0.78 & $-0.0120(-0.0228,-0.011), 0.031^{*}$ & -22.76 \\
\hline 5 and above & $-0.0001(-0.0003,-0.00003), 0.017^{*}$ & -0.28 & $-0.0229(-0.038,-0.0079), 0.003 *$ & -43.70 \\
\hline \multicolumn{5}{|c|}{ Age of children } \\
\hline $6-11$ & Ref. & & Ref. & \\
\hline $12-23$ & $-0.0001(-0.0001,-0.00002), 0.011^{*}$ & -0.14 & $0.0007(-0.0165,0.0179), 0.939$ & 1.34 \\
\hline $24-59$ & $0.0046(0.0039,0.0053),<0.001^{*}$ & 8.83 & $0.0119(-0.3450,0.0588), 0.618$ & 22.70 \\
\hline \multicolumn{5}{|c|}{ Birth size of children } \\
\hline Small & Ref. & & Ref. & \\
\hline Average & $-0.0006(-0.001,-0.0002), 0.003 *$ & -1.10 & $-0.0151(-0.0347,0.0044), 0.129$ & -28.84 \\
\hline Large & $0.0003(0.0001,0.0005), 0.003 *$ & 0.57 & $-0.0207(-0.0366,-0.0049), 0.010^{*}$ & -39.51 \\
\hline \multicolumn{5}{|l|}{ Fever } \\
\hline No & Ref. & & Ref. & \\
\hline Yes & $-0.0016(-0.0027,-0.0005), 0.005^{*}$ & -2.98 & $0.0097(0.003,0.0191), 0.043^{*}$ & 18.45 \\
\hline \multicolumn{3}{|l|}{ Constant } & $0.1527(0.0543,0.2510), 0.002^{*}$ & 290.74 \\
\hline
\end{tabular}

\section{Determinants of infant mortality}

Model comparison

Table 5 Model comparison between standard logistic regression and mixed-effects logistic regression models

\begin{tabular}{|l|l|l|l|}
\hline Model & AIC & BIC & Deviance \\
\hline Logistic regression model & 8429.103 & 8593.054 & 8381.103 \\
\hline Mixed-effect logistic regression model & 8160.937 & 8331.718 & 8110.94 \\
\hline
\end{tabular}

Table 6 Random effect parameters for mixed-effects logistic regression model

\begin{tabular}{|l|l|l|}
\hline Random effects parameters & Null model & Full model \\
\hline Cluster variance & $0.319(0.125,0.819)$ & $0.230(0.085,0.625)$ \\
\hline ICC & $0.089(0.035,0.199)$ & $0.065(0.025,0.160)$ \\
\hline MOR & 1.71 & 1.56 \\
\hline PCV & Ref. & 0.279 \\
\hline
\end{tabular}

The mixed-effect binary logistic regression model was the best-fitted model because it had a lower deviance 
value (Table 5). The ICC value was 0.065 (95\% CI: $0.025,0.160)$, which is indicates that $6.5 \%$ of the total variability in prevalence of anemia among children aged between 6 and 59 months is significantly attributable to the regional level and remaining $93.5 \%$ is attributable to individual levels or with in region difference. the LR test was $\left(X^{2}=778.41\right.$ with $\mathrm{P}$-value $\left.<0.001\right)$ which tells mixed-effect binary logistic regression model was the best-fitted model. Furthermore, the MOR-value in the full model was 1.56, indicates that children in high anemia prevalence clusters were 1.56 times higher likelihood of anemic compared to children in low anemia prevalence clusters (Table 6). So interpretation and reports were made based on this model. Of all explanatory variables included in the full model for multilevel analysis, mothers' age, source of drinking water, number of under-five children in the household, sex of household head, wealth status of the household, anemia level of mother, birth order of children, being single or twin, age of children, birth size of children, fever in the last two week prior to the survey, cough in the last two week prior to the survey, stunting, underweight and wasting were significantly associated with under-five children anemia in Ethiopia. The odds of anemia among children aged between 6 and 59 months from families who are not got improved water were increased by $16.5 \%$, as compared to those from families who are got improved water access. The odds of having anemic among children aged between aged 6 and 59 months from families having 4 and more under-five children were $46.3 \%$ (AOR $=1.463 ; 95 \%$ CI: $1.041-$ 2.058) higher than those from families having 3 and less under-five children. Households headed by female, the odds of anemic among children aged 6 to 59 months were $32.5 \%(\mathrm{AOR}=1.325 ; 95 \% \mathrm{CI}$ : $1.115-1.576)$ higher than children whose families were headed by male. Being single, the odds of having anemia were $66.5 \%$ (AOR $=0.665 ; 95 \%$ CI: $0.482-0.918$ ) lower than those children who were twin. The odds of having anemia among children whose birth size was big is $91.8 \%(\mathrm{AOR}=0.918 ; 95 \% \mathrm{CI} 0.802-1.051)$ lower as compared to children whose birth size was small at birth. The odds of developing anemia among children in the age group between 12 and 23 months were 0.606 times $(\mathrm{AOR}=0.606$; 95\%CI: $0.498-0.737)$ lower than those children whose age was between 6 and 11 months. The odds of anemia among children aged between 6 and 59 months born to mothers aged $24-34$ years and $35-49$ years were decreased by $84.4 \%(\mathrm{AOR}=0.844 ; 95 \% \mathrm{CI}: 0.0 .719-0.990)$ and $68.6 \%(\mathrm{AOR}=0.686 ; 95 \% \mathrm{CI}: 0.557-0.844)$ as compared to those born to mothers aged $<23$ years respectively. The odds of anemia prevalence among children aged 6 to 59 months who were normal (average) were $0.837(\mathrm{AOR}=0.837 ; 95 \% \mathrm{CI}: 0.737-0.956)$ times lower than those children who were small size at birth. The odds of anemia among children aged between 6 and 59 months who are from families having wealth status of lower, middle, riche and richest family were 0.669 (AOR $=0.669 ; 95 \% \mathrm{CI}: 0.575-0.779), 0.561(\mathrm{AOR}=$ $0.561 ; 95 \% \mathrm{CI}: 0.480-0.956), 0.616), 0.616(\mathrm{AOR}=0.616$; 95\% CI: $0.523-0.729)$ and $0.482(\mathrm{AOR}=0.482$; $95 \% \mathrm{CI}: 0.401-0.580)$ times lower than among children aged between 6 and 59 months who are from families having lowest wealth status (Table 7).

Table 7 Bi-variable and multivariable mixed-effect logistic regression analysis of determinants of anemia prevalence among children aged between 6 and 59 months in Ethiopia, 2016

\begin{tabular}{|c|c|c|c|c|c|}
\hline Variable & Characteristics & COR (95\% CI) & P-value & AOR (95\% CI) & P-value \\
\hline \multirow[t]{3}{*}{ Age of mother } & $15-23$ & Ref. & & Ref. & \\
\hline & $24-34$ & $0.811(0.716,0.919)$ & $0.001^{*}$ & $0.844(0.719,0.990)$ & $0.038^{*}$ \\
\hline & $35-49$ & $0.645(0.559,0.744)$ & $<0.001^{*}$ & $0.686(0.557,0.844)$ & $<0.001^{*}$ \\
\hline \multirow[t]{2}{*}{ source of water } & Not Improved & Ref. & & Ref, & \\
\hline & Improved & $0.920(0.835,1.101)$ & 0.09 & $1.165(1.052,1.291)$ & $0.003^{*}$ \\
\hline \multirow{2}{*}{$\begin{array}{l}\text { NO. under } 5 \\
\text { Children }\end{array}$} & $1-3$ & Ref. & & Ref. & \\
\hline & 4 and above & $2.126(1.521,2.972)$ & $<0.001^{*}$ & $1.463(1.041,2.056)$ & $0.028^{*}$ \\
\hline \multirow[t]{2}{*}{ Sex of HHH } & Male & Ref. & & Ref. & \\
\hline & Female & $1.359(1.189,1.552)$ & $<0.001 *$ & $1.325(1.115,1.576)$ & $0.001^{*}$ \\
\hline \multirow{5}{*}{ Wealth Index } & Lowest & Ref. & & Ref. & \\
\hline & Lower & $0.574(0.498,0.661)$ & $<0.001 *$ & $0.669(0.575,0.779)$ & $<0.001^{*}$ \\
\hline & Middle & $0.455(0.391,0.528)$ & $<0.001 *$ & $0.561(0.480,0.656)$ & $<0.001^{*}$ \\
\hline & Higher & $0.441(0.372,0.517)$ & $<0.001 *$ & $0.616(0.523,0.729)$ & $<0.001^{*}$ \\
\hline & Highest & $0.446(0.387,0.513)$ & $<0.001^{*}$ & $0.482(0.401,0.580)$ & $<0.001^{*}$ \\
\hline \multirow{2}{*}{$\begin{array}{l}\text { Mother anemia } \\
\text { level }\end{array}$} & Not anemic & Ref. & & Ref. & \\
\hline & Anemic & $2.076(1.867,2.307)$ & $<0.001 *$ & $1.664(1.487,1.862)$ & $<0.001^{*}$ \\
\hline \multirow{4}{*}{ Birth order } & First & Ref. & & Ref. & \\
\hline & Second & $0.840(0.731,0.965)$ & $0.014^{*}$ & $0.780(0.641,0.949)$ & $0.013^{*}$ \\
\hline & Three to four & $0.819(0.711,0.943)$ & $0.006^{*}$ & $0.831(0.698,0.989)$ & $0.037^{*}$ \\
\hline & 5 and above & $0.942(0.834,1.064)$ & 0.338 & $0.893(0.778,1.025)$ & 0.106 \\
\hline \multirow[t]{2}{*}{ Birth type } & Single & $0.999(0.731,1.365)$ & 0.19 & $0.665(0.482,0.918)$ & $0.013 *$ \\
\hline & Multiple & Ref. & & Ref. & \\
\hline
\end{tabular}




\begin{tabular}{|l|l|l|l|l|l|}
\hline \multirow{2}{*}{$\begin{array}{l}\text { Age of child in } \\
\text { months }\end{array}$} & $6-11$ & Ref. & & Ref. & \\
\cline { 2 - 6 } & $12-23$ & $0.747(0.615,0.911)$ & $<0.004^{*}$ & $0.606(0.498,0.737)$ & $<0.001^{*}$ \\
\cline { 2 - 5 } & $24-59$ & $0.323(0.272,0.385)$ & $<0.001^{*}$ & $0.249(0.206,0.294)$ & $<0.001^{*}$ \\
\hline \multirow{3}{*}{ Birth Size } & Small & Ref. & & Ref. & \\
\cline { 2 - 6 } & Average & $0.721(0.638,0.815)$ & $<0.001^{*}$ & $0.837(0.737,0.950)$ & $0.006^{*}$ \\
\cline { 2 - 6 } & Large & $0.687(0.603,0.783)$ & $<0.001^{*}$ & $0.918(0.802,1.051)$ & 0.217 \\
\hline \multirow{2}{*}{ Fever } & No & Ref. & & Ref. & \\
\cline { 2 - 6 } & Yes & $1.397(1.212,1.610)$ & $<0.001^{*}$ & $1.427(1.211,1.683)$ & $<0.001^{*}$ \\
\hline \multirow{2}{*}{ Cough } & No & Ref. & & Ref. & \\
\cline { 2 - 6 } & Yes & $1.167(1.024,1.330)$ & $0.021^{*}$ & $0.864(0.749,0.997)$ & $0.046^{*}$ \\
\hline \multirow{2}{*}{ Underweight } & No & Ref. & & Ref. & \\
\cline { 2 - 6 } & Yes & $1.294(1.171,1.429)$ & $<0.001^{*}$ & $1.535(1.359,1.729)$ & $<0.001^{*}$ \\
\hline & Ye & Ref. & & Ref. & \\
\hline \multirow{2}{*}{ Wasting } & No & $1.530(1.368,1.712)$ & $<0.001^{*}$ & $1.200(1.040,1.384)$ & $<0.001^{*}$ \\
\cline { 2 - 6 } & Yes & Ref. & & Ref. & \\
\hline
\end{tabular}

\section{Discussion}

Severity levels of anemia among children aged 6 - 59 months in Ethiopia was a major public health problem. In this study, the trend of anemia prevalence has been significantly increase from $44.5 \%$ in 2005 to $57.2 \%$ in 2016 (overall phase). This finding is coincide with the study done in Sub - Saharan Africa and Lao People's Democratic Republic $[4,27]$ respectively. The reason for the high prevalence of anemia in 2016 compared to the previous EDHS reports could be linked to the drought that affected crop and milk production in most parts of Ethiopia in 2015 and 2016, further Ethiopian has not achieved the national nutrition strategy, which is aimed at reducing the prevalence of childhood anemia by $30 \%$ and $20 \%$ in 2015 and 2020 , respectively [27]. When we decompose this change, behavioral change of the respondents between the surveys contributed $47.1 \%$ for the increment of anemia prevalence over the last eleven years and the remaining $52.87 \%$ of increment was due to difference in characteristics (population proportion). Behavioral change of households who had lowest wealth status were contributed $1.68 \%$ for the increment of anemia prevalence for the last eleven years as compared to households who had highest wealth status. Similarly, the behavioral households having four and more than four under-five children contributing $2.0 \%$ for the increment of anemia prevalence for the last eleven years as compared to households having less than four under-five children. It is higher than the study [38] in Brazil and [39] in Ecuador. The possible reason may be due to the long-standing prevalence of severe malnutrition among under-five children, because of insufficient dietary intake of nutrients, in sub-Saharan Africa [40]. Besides, SubSaharan African children are highly affected by infectious diseases such as malaria, hookworms, Schistosoma, and visceral leishmaniasis, due to their everyday exposure to poor cleanliness and environmental circumstances that favor the transmission and spread of parasites [41-43].

Furthermore, in the mixed binary logistic regression model, we found that maternal age, source of drinking water, number of under-five children, sex of household head, wealth status, anemia level of mother, birth order of children, birth type of children, age of children, birth size of children, fever, cough, stunting, underweight and wasting taking drugs for the intestinal parasite in the last six months, and sub-Saharan African region were significantly associated the prevalence of anemia among children aged between 6 and 59 months.

In this finding, the odds of being anemic were lower for children whose households used improved source of drinking water. This study is consistent with $[18,26]$ and Ethiopian and Gahanna respectively. Precarious sanitation conditions are linked with anemia, even if indirectly. Since such conditions are associated with greater number of infectious and parasitic diseases, which in turn contributed towards diminishing the hemoglobin levels which is associated with higher rates of infectious diseases, although presence of fever, cough, diarrhea malaria which was associated with water access and are responsible for the increment of anemia prevalence.

Children of the rich and middle class households had lower prevalence of anemia compared to the poorest households. This study is compatible with the study [12, 22] in Ethiopia, [4] in Sub- Saharan Africa, [25] in Bangladesh and [26] in Ghana. The possible reason might be because poverty is strongly related with food insecurity and hereafter children from households with lower income household may not have an access to foods rich in iron, vitamin, and folic acid, which in go increases their risk of developing anemia. The other possible explanation is that families with low income are less likely to obtaining nutrient-rich foods, secure food availability, and afford health services during illness for their children.

Maternal anemia was significantly associated with higher odds of anemia prevalence among children aged between 6 and 35 months. It is coinciding with the study findings [12, 24] in Ethiopia [44] in Togo. This may be due to the mother is the primary source of food for children and the children share a similar diet, so their eating 
habits and quality of life could be identical. Also, through transplacental transmission and breastfeeding, infectious causes of anemia such as malaria and HIV/AIDS that can interfere with their development of red blood cells and iron stores may be transferred to childhood. The results also showed that the likelihood of children in the age range of 6 to 11 months being anemic was higher than those in the age range of 12 to 23 and 34 to 59 months. The result of the study was consistent with the result of various studies [18] in Ethiopia, [8] in Uganda and [26] in Ghana. One of the possible explanations for the higher likelihood of anemia might be related to low balanced nutritional intakes that may not be sufficient for their rapid growth related demands. The other possible reasons might be due to the fact that poor infant and young children feeding practices particularly timely initiation of complementary food is low in Ethiopia as evidenced by published data of 2005 and 2011 EDHS survey [45].

Children with a history of cough and fever had higher odds of higher levels of anemia compared to children who did not have cough and fever, respectively. It is consistent with studies reported [4] in Sub-Saharan Africa, [12] in Ethiopia and [26]in Ghana. This could be due to children with febrile and cough sickness might have a loss of appetite, and reduced absorption of necessary nutrients (iron, folate, and vitamin B12) that might rise the possibility of anemia [46]. Besides, the presence of cough and fever might indicate the occurrence of infectious diseases such as visceral leishmaniasis, malaria, hookworm, ascariasis, giardiasis, and amoebiasis, which are the leading causes of anemia in children [47, 48].

In this study, the odds of prevalence of anemia for stunted, underweight and wasted children aged between 6 and 59 months were higher than normal children. This was consistent with studies [4]in Sun-Saharan Africa and [12] in Ethiopia. The reason behind it is, poor nutritional status is associated with poor immunity and, hence, infections and infestations also have synergistic effects of micronutrient shortages for prevalence of anemia [40]. Also, undernourished children are disposed to micronutrient shortages, such as iron, vitamin A, vitamin B12 and folic acid, which are helpful for haemoglobin and DNA synthesis during red blood cell production, and in turn, results in anemia [49]. Anemia and malnutrition often share common causes; it is likely that multiple types of malnutrition would co-occur in the same individuals, and increase the improvement of anemia in synergetic manner. Further, the gastrointestinal epithelium trouble in undernourished individuals may decrease absorption, and contribute towards expansion and worsening of anemia [50]. Accordingly, low hemoglobin level may also compromise the linear growth of the children [51].

Additionally, the results indicated that the higher anemia status was more likely for children from households with greater number of children under the age of five years in Ethiopia. This agreed with the result of other study done [20] in Bangladesh. The possible reason is due to the fact that the household with greater number of children has high demand and expenditure for food to satisfy the needs of members of the family. In conjunction, this may worsen the quality of care for children, and increase the risk of anemia not only for children but also for adults.

Mother's age was significantly associated with anemia among children. Children from ( $24-34$ years old) and $(35-49$ years old) mothers were less likely to be anemic compared to children from young women $(15-23$ years old). This finding was in line with a study [4].The reason for this might be poor child care practice among young mothers.

This finding showed that being twin were more risk to have anemia as compared to children who were single. This finding is consistent with the study conducted [25] in Bangladesh and [24] in Ethiopia. This might be due to children who are twin might not get exclusive breast milk at early ages and this reduce their immunity and prone to diarrhea. Similarly, the quality of care and attention from parents decreased. So they are easily susceptible for different diseases.

\section{Strength and limitations}

This study had numerous strengths. First, the study was based on nationally representative big datasets, and therefore it had adequate statistical power. Second, the estimates of the study were done after the data were weighted for the probability sampling and nonresponse, to make it representative at national and regional levels: therefore, it can be generalized to all births from reproductive-age women in Ethiopia. Third, multivariate decomposition analysis was adopted to understand the factors that significantly contributed to the increase the prevalence of childhood anemia over time. Limitation, since this study was based on secondary data, we were not able to investigate all factors that may be relevant to anemia in children, including eating habits, parasite infestations (malaria, Visceral Leishmaniasis, and hookworm, previous hospitalization, and use of nutritional supplements (such as vitamin B12 and folate). Furthermore, variables such as the birth size of a child which is the subjective measurement of the birth size of a child were included in this study since the measured birth weight was not found for the majority of the children so this might overestimate or underestimate the effect size of birth size. 


\section{Conclusion and Policy Recommendation}

In conclusion, anemia among children aged 6-59 months in Ethiopia was a major public health problem. maternal age, type of birth, fever and cough in the last two weeks, stunted, wasted, underweight, child age, birth order, household wealth status, family size, maternal anemia and sex of household head in Ethiopia were found significant determinants of the prevalence of childhood anemia. Interventions to address maternal anemia, febrile illness and cough disease as well as and strengthening the economic status of the family are recommended to reduce the prevalence of childhood anemia. Moreover, it is better to strengthen the strategies of early recognition and organization of stunted, wasted, and underweight children to decrease the prevalence childhood anemia.

\section{Abbreviations}

AOR: Adjusted Odds Ratio, AIC: Akakie Information Criteria, Cofe: Coefficient, COR: Crude Odds Ratio, CSA: Central Statistical Agency; BIC: Bayesian Information Criteria, DHS: Demographic health survey; EAs: Enumeration areas, EDHS: Ethiopian demographic and health survey, ICC: Intra-cluster Correlation Coefficient, LLR: Log likelihood ratio, LR: Likelihood ratio; MDG: Millennium Development Goals, MOR: Median Odds Ratio, PCV: Proportional Change in Variance, SNNP: Southern Nations and Nationalities of People, SSA: SubSaharan Africa, Ref.: Reference Category.

\section{Acknowledgments}

We would like to thank the measure DHS program for providing the data set.

Conflict of interests

The authors declare that they have no competing interests.

\section{Authors' contributions}

AA and YA were involved for this study from the beginning to design, acquisition of data, data washing, data analysis and interpretation and recruiting and reviewing of the manuscript. AA prepared the final draft of the manuscript. Both authors read and approved the final manuscript.

\section{Funding}

We did not receive any fund for this research.

\section{Availability of data and materials}

The Ethiopian demographic health survey data sets are open and can be accessed from the Measure DHS website (https://www.dhsprogram.com/data/dataset admin/login main.cfm). Through an online request by explaining the objective of the study. The datasets analyzed during the study are available from the corresponding author upon reasonable request.

\section{Ethics approval and consent to participate}

Ethical clearance for the survey (2000-2016) was provided by the Ethiopian Health and Nutrition Research Institute (EHNRI) Review Board and the National Research Ethics Review Committee (NRERC) at the Ministry of Science. Respondents were informed about the survey and verbal consent was taken. The detail about ethical approval and consent to participate available in the EDHS's report [52].

\section{Consent for publication}

Not applicable.

\section{Reference}

1. SPRING, Understanding anemia: Guidance for conducting a landscape analysis. 2017.

2. WHO., Global anaemia reduction efforts among women of reproductive age: impact, achievement of targets and the way forward for optimizing efforts. 2020: Geneva.

3. Kejo, D., et al., Prevalence and predictors of anemia among children under 5 years of age in Arusha District, Tanzania. Pediatric health, medicine and therapeutics, 2018. 9: p. 9.

4. Tesema, G.A., et al., Prevalence and determinants of severity levels of anemia among children aged 6-59 months in sub-Saharan Africa: A multilevel ordinal logistic regression analysis. PloS one, 2021. 16(4): $p$. e0249978.

5. Gebreegziabiher, G., B. Etana, and D. Niggusie, Determinants of anemia among children aged 6-59 months living in Kilte Awulaelo Woreda, northern Ethiopia. Anemia, 2014. 2014.

6. Khulu, C. and S. Ramroop, Key Determinants of Anemia among Youngsters under Five Years in Senegal, Malawi, and Angola. International Journal of Environmental Research and Public Health, 2020. 17(22): p. 8538.

7. Moschovis, P.P., et al., Individual, maternal and household risk factors for anaemia among young children in sub-Saharan Africa: a cross-sectional study. BMJ open, 2018. 8(5).

8. Kuziga, F., Y. Adoke, and R.K. Wanyenze, Prevalence and factors associated with anaemia among children aged 6 to 59 months in Namutumba district, Uganda: a cross-sectional study. BMC pediatrics, 2017. 17(1): p. 1-9. 
9. Al-Jawaldeh, A., et al., Are Countries of the Eastern Mediterranean Region on Track towards Meeting the World Health Assembly Target for Anemia? A Review of Evidence. International Journal of Environmental Research and Public Health, 2021. 18(5): p. 2449.

10. Dirren, H., et al., Altitude correction for hemoglobin. European journal of clinical nutrition, 1994. 48(9): p. 625-632.

11. Organization, U.N.W.H., Iron Deficiency Anaemia: Assesment, Prevention and Control: a Guide for Programme Managers. 2001: World Health Organization.

12. Asresie, M.B., G.A. Fekadu, and G.W. Dagnew, Determinants of anemia among children aged 6-59 months in Ethiopia: Further analysis of the 2016 Ethiopian demographic health survey. Advances in Public Health, 2020. 2020.

13. Organization, W.H., Iron deficiency anemia. assessment, prevention, and control. A guide for programme managers, 2001: p. 47-62.

14. Ogunsakin, R.E., B.T. Babalola, and O. Akinyemi, Statistical Modeling of Determinants of Anemia Prevalence among Children Aged 6-59 Months in Nigeria: A Cross-Sectional Study. Anemia, 2020. 2020.

15. Sanou, D., H. Turgeon-O'Brien, and T. Desrosiers, Prévalence et déterminants non alimentaires de l'anémie et de la carence en fer chez des orphelins et enfants vulnérables d'âge préscolaire du BurkinaFaso. Nutrition clinique et mátabolisme, 2008. 22(1): p. 10-19.

16. Lozoff, B., E. Jimenez, and A.W. Wolf, Long-term developmental outcome of infants with iron deficiency. New England journal of medicine, 1991. 325(10): p. 687-694.

17. Pollitt, E., Iron deficiency and cognitive function. Annual review of nutrition, 1993. 13(1): p. 521-537.

18. Muchie, K.F., Determinants of severity levels of anemia among children aged 6-59 months in Ethiopia: further analysis of the 2011 Ethiopian demographic and health survey. BMC Nutrition, 2016. 2(1): p. 1-8.

19. CSA, Ethiopian Demographic and Health Survey 2016.

20. Khan, J.R., N. Awan, and F. Misu, Determinants of anemia among 6-59 months aged children in Bangladesh: evidence from nationally representative data. BMC pediatrics, 2016. 16(1): p. 1-12.

21. Jembere, M., R.H. Kabthymer, and A. Deribew, Determinants of Anemia Among Children Aged 6 to 59 Months in Dilla Town, Southern Ethiopia: A Facility Based Case Control Study. Global Pediatric Health, 2020. 7: p. $2333794 X 20974232$.

22. Chernet, A.G., T. Nega, and M.D. Biru, Prevalence and Associated Factors of Anaemia severity among Children in Ethiopia. vol.

23. Yusuf, A., et al., Factors influencing childhood anaemia in Bangladesh: a two level logistic regression analysis. BMC pediatrics, 2019. 19(1): p. 1-9.

24. Mohammed, S.H., T.D. Habtewold, and A. Esmaillzadeh, Household, maternal, and child related determinants of hemoglobin levels of Ethiopian children: hierarchical regression analysis. BMC pediatrics, 2019. 19(1): p. 1-10.

25. Rahman, M.S., et al., Association between malnutrition and anemia in under-five children and women of reproductive age: Evidence from Bangladesh Demographic and Health Survey 2011. PloS one, 2019. 14(7): p. e0219170.

26. Shenton, L.M., A.D. Jones, and M.L. Wilson, Factors associated with anemia status among children aged 6-59 months in Ghana, 2003-2014. Maternal and child health journal, 2020. 24(4): p. 483-502.

27. Keokenchanh, S., et al., Prevalence of anemia and its associated factors among children aged 6-59 months in the Lao People's Democratic Republic: A multilevel analysis. Plos one, 2021. 16(3): p. e0248969.

28. Gebereselassie, Y., et al., Magnitude, Severity, and Associated Factors of Anemia among Under-Five Children Attending Hawassa University Teaching and Referral Hospital, Hawassa, Southern Ethiopia, 2016. Anemia, 2020. 2020.

29. Simbauranga, R.H., et al., Prevalence and factors associated with severe anaemia amongst under-five children hospitalized at Bugando Medical Centre, Mwanza, Tanzania. BMC hematology, 2015. 15(1): p. 19.

30. Gebreweld, A., et al., Prevalence of anemia and its associated factors among children under five years of age attending at Guguftu health center, South Wollo, Northeast Ethiopia. PloS one, 2019. 14(7): p. e0218961.

31. Enawgaw, B., et al., Prevalence of anemia and associated factors among hospitalized children attending the University of Gondar Hospital, Northwest Ethiopia. EJIFCC, 2019. 30(1): p. 35.

32. Gebremeskel, M.G. and L.L. Tirore, Factors Associated with Anemia Among Children 6-23 Months of Age in Ethiopia: A Multilevel Analysis of Data from the 2016 Ethiopia Demographic and Health Survey. Pediatric Health, Medicine and Therapeutics, 2020. 11: p. 347.

33. CSA, Ethiopian Demographic and Health Survey 2005.

34. CSA, Ethiopian Demographic and Health Survey 2011.

35. Kawo, K.N., Z.G. Asfaw, and N. Yohannes, Multilevel analysis of determinants of anemia prevalence 
among children aged 6-59 months in Ethiopia: classical and Bayesian approaches. Anemia, 2018. 2018.

36. Little, R.J. and D.B. Rubin, Statistical analysis with missing data. Vol. 793. 2019: John Wiley \& Sons.

37. Powers, D.A., H. Yoshioka, and M.-S. Yun, mvdcmp: Multivariate decomposition for nonlinear response models. The Stata Journal, 2011. 11(4): p. 556-576.

38. De Oliveira, J., et al., Iron deficiency anemia in children: prevalence and prevention studies in Ribeir ão Preto, Brazil. Archivos latinoamericanos de nutricion, 1997. 47(2 Suppl 1): p. 41-43.

39. Quizhpe, E., et al., Prevalence of anaemia in schoolchildren in the Amazon area of Ecuador. Revista panamericana de salud publica= Pan American journal of public health, 2003. 13(6): p. 355-361.

40. Bhutta, Z.A., et al., Severe childhood malnutrition. Nature reviews Disease primers, 2017. 3(1): p. 1-18.

41. Smith, H., Prospects for the control of neglected tropical disease by mass drug administrative. Expert Review of Anti-Infective Therapy, 2009. 7(1): p. 218.

42. Fineberg, H. and M. Wilson, Emerging infectious diseases. IRGC Report, 2010.

43. Greenwood, B., The epidemiology of malaria. Annals of Tropical Medicine \& Parasitology, 1997. 91(7): p. 763-769.

44. Nambiema, A., A. Robert, and I. Yaya, Prevalence and risk factors of anemia in children aged from 6 to 59 months in Togo: analysis from Togo demographic and health survey data, 2013-2014. BMC public health, 2019. 19(1): p. 1-9.

45. Disha, A., et al., Factors associated with infant and young child feeding practices in Amhara region and nationally in Ethiopia: analysis of the 2005 and 2011 demographic and health surveys. Washington, DC: Alive and Thrive, 2015.

46. Semrad, C.E., Approach to the patient with diarrhea and malabsorption. Goldman's cecil medicine, 2012: $\mathrm{p}$. 895.

47. van den Bogaart, E., et al., Prevalence, features and risk factors for malaria co-infections amongst visceral leishmaniasis patients from Amudat Hospital, Uganda. PLoS neglected tropical diseases, 2012. 6(4): p. e1617.

48. Le, H.T., et al., Anemia and intestinal parasite infection in school children in rural Vietnam. Asia Pacific Journal of Clinical Nutrition, 2007. 16(4).

49. Ahmed, T., M. Hossain, and K.I. Sanin, Global burden of maternal and child undernutrition and micronutrient deficiencies. Annals of Nutrition and Metabolism, 2012. 61(Suppl. 1): p. 8-17.

50. Pasricha, S.-R., Anemia: a comprehensive global estimate. Blood, The Journal of the American Society of Hematology, 2014. 123(5): p. 611-612.

51. Soliman, A.T., et al., Linear growth in children with iron deficiency anemia before and after treatment. Journal of tropical pediatrics, 2009. 55(5): p. 324-327.

52. survey, E.E.d.a.h., key indicators report. 2016( The DHS Program ICF. 2016). 